03,09, 12

\title{
Резонансное рассеяние света оптическими фононами в гомоэпитксиальном нанослое $n$-GaP, выращенном на подложке (001)n-GaP
}

\author{
(C) Б.Х. Байрамов \\ Физико-технический институт им. А.Ф. Иоффре \\ Санкт-Петербург, Россия \\ E-mail: bairamov@mail.ioffe.ru
}

Поступила в Редакцию 12 октября 2020 г.

В окончательной редакции 12 октября 2020 г.

Принята к публикации 13 октября 2020 г.

\begin{abstract}
Представлены результаты обнаружения резонансного усиления интенсивности рассеяния света оптическими фононами в гомоэпитаксиальном наномасштабном слое $n$-GaP, толщиной $70 \mathrm{~nm}$, выращенном методом газофазной эпитаксии из металлоорганических соединений на проводящей сильнолегированной подложке кристалла $n$ - GaP, ориентированной по оси (001). Показано, что при комнатной температуре в спектре рамановского рассеяния света такого нанослоя $(001) n-\mathrm{GaP}$ в образце $n-\mathrm{GaP} / n-\mathrm{GaP}(001)$ в сравнении со спектром высокоомного кристаллического образца (001) si-GaP в диапазоне частот от 600 до $800 \mathrm{~cm}^{-1}$ удается обнаружить достаточно узкие полосы линий рассеяния света второго порядка. Установлено, что такие полосы обусловлены суммарными комбинациями и обертонами поперечных $\mathrm{TO}(\Gamma)$ и продольных $\mathrm{LO}(\Gamma)$ оптических фононов, с волновыми векторами соответствующими точкам $\Sigma, \mathrm{K}, \mathrm{X}, \mathrm{L}$ и Г зоны Бриллюэна кристалла GaP. Показано, что рассеяние света носит резонансный характер, и обусловлено присутствием примесей вследствие проявления экситон-фононного взаимодействия.
\end{abstract}

Ключевые слова: гомоэпитаксиальный наномасштабный слой (001) n-GaP, сильнолегированная подложка, резонансное двухфононное рассеяние.

DOI: 10.21883/FTT.2021.02.50465.218

\section{1. Введение}

Исследования физических свойств гомоэпитаксиальных слоев (включая и наномасштабной толщины) широкозонных полупроводников на исходных сильнолегированных подложках того же материала привлекают повышенный интерес, поскольку они демонстрируют в сравнении с гетероэпитаксиальными слоями лучшую совокупность физических свойств - высокую степень кристаллического совершенства структуры с меньшей плотностью дислокаций и других структурных дефектов, оптических, электрических и иных свойств. Совершенные и высококачественные гетероэпитаксиальные слои выращиваются только при определенных условиях послойного роста, происходящего через зарождение на поверхности и рост двумерных островков новой фазы, рассматриваемого как 2D фазовый переход первого рода, и их последующий рост до образования полного монослоя растущего монокристалла [1-9]. Безусловно такие привлекательные особенности гомоэпитаксиальных слоев вызывают необходимость развития и максимально эффективных методов исследования их кристаллической структуры и электрических свойств. При этом, на пути создания приборных структур на основе гомоэпитаксиальных слоев, выращенных на сильнолегированных подложках являются очевидные сложности определения степени совершенства кристаллической структуры, а также исследования их электрических свойств традици- онными времязатратными методами, требующими приготовления омических контактов и проведения электрических измерений проводимости и эффекта Холла $[5,8,9]$.

Резонансная спектроскопия рамановского рассеяния света является одним из наиболее привлекательных методов исследования кристаллической структуры и динамики элементарных решеточных и электронных возбуждений в легированных полупроводниковых материалах [10-40]. При этом, реальные промежуточные электронные состояния играют ключевую роль, формируя квантовые пути, определяющие природу важных механизмов нерезонансного и резонансного поведения рассеяния, а также квантовой интерференции в возбуждающем и рассеянном каналах процесса рассеяния света.

Впервые теория однофононного резонансного комбинационного рассеяния света (РКРС), учитывающая экситонные эффекты была дана Лоудоном [11]. В [12,13] было получено выражение для тензора резонансного КРС при учете слабого экситон-фотонного взаимодействия для механизмов деформационного потенциала и фрелиховского электрон-фононного взаимодействия. Аналогичные расчеты были выполнены в работе [14] с использованием функций Грина. При этом учитывалась рассеяние только через одну валентную зону и зону проводимости. Такая модель резонансного рассеяния не учитывает кулоновского взаимодействия промежуточными электронными состояниями, рассматривая их как состояния несвязанной электрон-дырочной пары. 
На основе экспериментальной возможности выполнения строгих количественных измерений интенсивностей для хорошо спектрально разрешенных узких линий акустических фононов [15], было установлено, что полученные экспериментальные данные зависимости интенсивности рассеяния света от энергии квантов возбуждающего излучения не достаточно хорошо описываются двухзонной теорией Лоудона [13]. Такой учет только одной валентной зоны оказался недостаточным для количественной интерпретации экспериментальных результатов по рассеянию света акустическими и оптическими фононами в кристаллах со структурой алмаза и цинковой обманки [15-18].

Поэтому, для преодоления возникших противоречий в пионерских работах [15-18] была развита теория многозонного резонансного усиления интенсивности рассеяния света сначала акустическими фононами [1517], а затем и оптическими фононами [18]. При этом в качестве промежуточных электронных состояний были введены ранее не рассматривавшиеся атомоподобные экситонные состояния, принадлежащие как дискретным экситонным зонам, так и непрерывному спектру, а также высоко-лежащие валентные зоны.

Первоначально многозонный характер резонансного усиления интенсивности рассеяния света был предсказан и экспериментально установлен для процесса резонансного рассеяния света Мандельштама-Бриллюэна акустическими фононами на примере кристаллов $\mathrm{ZnSe}$ [15-17] с учетом экситонных корреляций как при внутризонных так и междузонных переходов между зоной проводимости $\Gamma_{6}$, четырехкратно вырожденными валентными зонами $\Gamma_{8}$ и двукратно вырожденной зоной $\Gamma_{7}$, отщепленной спин-орбитальным взаимодействием. При этом, в качестве промежуточных виртуальных электронных состояний, вовлеченных в процессы рассеяния, рассматривались реальные экситонные состояния ВаньеМотта, принадлежащие как дискретным водородоподобным экситонным зонам, так и непрерывному спектру. В дальнейшем развитый подход многозонного характера резонансного усиления интенсивности рассеяния света был подтвержден для рамановского рассеяния света на оптических фононах, на примере кристаллов AgI [18].

В последующие годы теория многозонного резонансного рассеяния света и развитые в [15-18] подходы были подтверждены и использованы в [19-22] и с их учетом были пересчитаны результаты ранее выполненных исследований по однофононному [19-21] и двухононному [22] резонансному рамановского рассеянию света оптическими фононами для ряда классических полупроводников соединений $\mathrm{A}^{3} \mathrm{~B}^{5}$ группы - GaP, GaAs, GaSb и InP, а также подтверждены для многих полупроводниковых материалов - от объемных до низкоразмерных наноструктур (например, [23-39] и многочисленными другими исследованиями, основывающимся на результатах использования подходов, разработанных в оригинальных работах [15-21]). Недавно, показано, что в кристаллах алмаза, легированных азотом, формирование большого числа электронных уровней, связанных с точечными азотно-вакансионными дефектами (nitrogen-vacancy NV center), обладающими уникальными спин-зависимыми свойствами, обусловило наиболее яркое проявление механизма многозонного резонансного неупругого рассеяния света оптическими фононами $[38,39]$.

В настоящей работе показано, что на основе развития спектроскопии микрорамановского рассеяния света может быть реализован метод резонансного усиления интенсивности рамановского рассеяния света второго порядка в гомоэпитаксиальном наномасштабном слое $n$-GaP, толщиной $70 \mathrm{~nm}$, выращенном на проводящей сильнолегированной подложке кристалла $n$-GaP, ориентированной по оси (001). Продемонстрировано, что такое рассеяние обусловлено обычно слабыми суммарными комбинациями и обертонами поперечных ТО(Г) и продольных $\mathrm{LO}(\Gamma)$ оптических фононов, с волновыми векторами $(\mathbf{k})$ соответствующими точкам $\Sigma, \mathrm{K}, \mathrm{X}, \mathrm{L}$ и Г зоны Бриллюэна кристалла $\mathrm{GaP}$ при оптическом возбуждении вблизи непрямого края поглощения легированного гомоэпитаксиального слоя $n$-GaP. При этом показано, что рассеяние света носит резонансный характер, и обусловлено присутствием примесей вследствие проявления экситон-фононного взаимодействия.

\section{2. Методика эксперимента}

Для наших исследований использовался образец n-GaP/n-GaP (001) с наномасштабным гомоэпитаксиальным слоем $n$-GaP толщиной $70 \mathrm{~nm}$, выращенным методом газофазной эпитаксии из металлоорганических соединений на проводящей сильнолегированной подложке кристалла $n$-GaP. Подложка ориентирована по оси (001) (образец № 1). Для непосредственного сравнения также использовался образец № 2 сильно компесированного высокоомного (удельное сопротивление $\sim 10^{12} \Omega \cdot \mathrm{cm}^{-1}$ ) полуизолирующего кристалла $s i$-GaP, также ориентированного по оси (001). Возбуждение спектров микро-рамановского рассеяния света осуществлялось излучением второй гармоники непрерывного лазера на алюмоиттриевом гранате с длиной волны $\lambda_{i}=532.070 \mathrm{~nm}$ по методике, приведенной в [33,35]. Спектральный состав рассеянного света регистрировался с помощью конфокального спектрометра HR 800 (Horiba Jobin Yvon), оснащенного микроскопом Olympus BX 41 с микрообъективом 100x (NA 0.9). Локальный размер возбуждающего пятна составлял $\sim 0.9 \mathrm{mkm}$. Все измерения спектров рамановского рассеяния выполнены в одних и тех же экспериментальных условиях для всех образцов при комнатной температуре и в конфигурации обратного рассеяния при параллельной поляризации падающего и рассеянного света $[Z(X X) Z-]$ с осями $X, Y$ и $Z$ соответственно, вдоль направлений $[1 \overline{1} 0],[110]$ и [001].

При этом, на пути создания приборных структур на основе гомоэпитаксиальных слоев, выращенных на сильнолегированных подложках появляются очевидные 
сложности определения степени совершенства кристаллической структуры, а также исследования их электрических свойств традиционными времязатратными методами, требующими приготовления омических контактов и проведения электрических измерений проводимости и эффекта Холла $[5,8,9]$.

\section{3. Экспериментальные результаты исследований и их обсуждение}

Как уже было отмечено, актуальными проблемами измерения структурных и электрических параметров гомоэпитаксиальных слоев, выращенных на сильнолегированных подложках, в частности, являются очевидные сложности комбинированного определения степени совершенства кристаллической структуры и основных параметров свободных носителей заряда - концентрации и подвижности на основе традиционного метода измерения электрических свойств полупроводниковых материалов путем выполнения электрических измерений проводимости и эффекта Холла [12]. В особенности такие сложности возникают для особо тонких гомоэпитаксиальных слоев наномасштабного уровня толщины.

Ранее было показано, что обнаружение в спектре рамановского рассеяния света в конфигурации обратного рассеяния при параллельной поляризации падающего и рассеянного света $[Z(X X) Z-]$, выявленном для гомоэпитаксиальныого слоя $n-\mathrm{GaP}$, толщиной $70 \mathrm{~nm}$ удалось экспериментально наблюдать достаточно узкие характерные линии рассеяния света первого порядка поперечными оптическими $\mathrm{TO}(\Gamma)$ - фононами при $365.50 \mathrm{~cm}^{-1}$ с полушириной (полной шириной на половине высоты (ПШПВ) равной $3.45 \mathrm{~cm}^{-1}$ [41]. Они были зарегистрированы в спектрах и для сильнолегированной подложки $n$-GaP (001) и полуизолирующего кристалла $s i$-GaP (001). Обнаруженные результаты позволили получить важную информацию о высоком совершенстве кристаллической структуры наномасштабного эпитаксиального слоя (001) $n$-GaP.

Кроме того, обнаружение и идентификация высокочастотной линии как обусловленной связанными продольными плазмон-фононными $\mathrm{LO}(\Gamma)_{+}$колебаниями при $407.22 \mathrm{~cm}^{-1}$ с полушириной $11.41 \mathrm{~cm}^{-1}$ для гомоэпитаксиального слоя $n$-GaP (001) и, соответственно, $406.74 \mathrm{~cm}^{-1}$ с полушириной $10.98 \mathrm{~cm}^{-1}$ для подложки $n$-GaP (001) в сравнении для продольных оптических $\mathrm{LO}(\Gamma)$ фононов при $402.30 \mathrm{~cm}^{-1}$ с полушириной $0.60 \mathrm{~cm}^{-1}$ ) для полуизолирующего кристалла $s i$-GaP (001), позволили определить локальную концентрацию $n$ и подвижность $\mu$ свободных носителей заряда для гомоэпитаксиального наномасштабного слоя $n$-GaP и подложки $n$-GaP (001). Расчеты выполнены на основе микроскопической модели рассеяния света $\mathrm{LO}(\Gamma)_{+}$колебаниями, обусловленного механизмами деформационного потенциала и электрооптическим рассеянием $[42,43]$. Полученные численные значения оказались равными $n_{\text {hepi }}=(3.25 \pm 0.1) \cdot 10^{17} \mathrm{~cm}^{3}$ и подвижности $\mu_{\text {hepi }}=(40.0 \pm 0.1) \mathrm{cm}^{2} \cdot \mathrm{V}^{-1} \cdot \mathrm{s}^{-1}$ для гомоэпитаксиального слоя (100) $n$-GaP в сравнении с $\quad n_{\text {subs }}=(2.52 \pm 0.1) \cdot 10^{17} \mathrm{~cm}^{-3} \quad$ и $\quad \mu_{\text {subs }}=$ $=(51.0 \pm 0.1) \mathrm{cm}^{2} \cdot V^{-1} \cdot \mathrm{s}^{-1}$ подложки $\quad(100) n-\mathrm{GaP}$ образца $n-\mathrm{GaP} / n-\mathrm{GaP}$ (100) [41].

Выявление высокого совершенства кристаллической структуры наномасштабного эпитаксиального слоя (001) $n$ - GaP и наличия примесей позволило обнаружить новые важные спектральные особенности в области двухфононного рассеяния образца $n-\mathrm{GaP} / n-\mathrm{GaP}$ (001). Из спектров рамановского рассеяния света, приведенных на рис. 1 , для образца № $1 \mathrm{GaP} / n-\mathrm{GaP}(001)$ хорошо видно, что в диапазоне частот от 600 до $800 \mathrm{~cm}^{-1}$, обнаруженном для гомоэпитаксиальныого слоя $n$-GaP, толщиной $70 \mathrm{~nm}$ GaP также удается обнаружить достаточно узкие полосы линий, соответствующие рассеянию света второго порядка оптическими фононами, при комнатной температуре. Такие же характерные линии зарегистрированы и в других спектрах - для сильнолегированной подложки $n$ - GaP (001) в образце $n$-GaP/n-GaP (001), а также и в отдельном образце полуизолирующего кристалла si-GaP (001) образец № 2. Все эти спектры получены в одних и тех же экспериментальных условиях при комнатной температуре и при возбуждении излучением лазера с длиной волны $\lambda_{i}=532.070 \mathrm{~nm} \mathrm{c}$ одинаковой плотностью мощности лазерного излучения.

Указанные на спектрах численные величины значения частоты и истинные полуширины линии - полная ширина на половине её высоты $\left(\mathrm{c} \mathrm{cm}^{-1}\right)$ получены из спектров рассеяния света первого порядка поперечными $\mathrm{TO}(\Gamma)-$ и продольными LO $(\Gamma)$ оптическими фононами, а также высокочастотными продольными связанными плазмон-фононными $\mathrm{LO}(\Gamma)_{+}$колебаниями с выделением лоренцевского контура из наблюдаемого фохтовского контура с учетом поправки на спектральное разрешение, описываемое гаусовским контуром. Эти значения частоты и истинные полуширины линий были использованы при расчётах, выполненных на основе микроскопической модели рассеяния света $\mathrm{LO}(\Gamma)_{+}$колебаниями, обусловленного механизмами деформационного потенциала и электрооптическим рассеянием [41] для определения концентраций и подвижностей свободных носителей заряда для гомоэпитаксиальныого наномасштабного слоя $n$-GaP и подложки $n$-GaP (001).

Спектр двухфононного рамановского рассеяния света в полуизолирующем кристалле $s i$-GaP (001), приведенный на рисунке хорошо согласуются с результатами исследований, выполненных ранее для возбуждения в области прозрачности кристалла специально нелегиро-

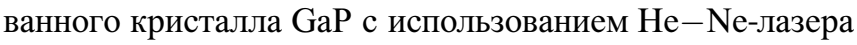
с длиной волны $\lambda_{i}=632.817 \mathrm{~nm}[40,41]$. Такое двухфононное рассеяние света обусловлено разрешенными правилами отбора для рассеяния света второго порядка и обычно слабыми по интенсивности (по сравнению с линиями $\mathrm{TO}(\Gamma)-$ и $\mathrm{LO}(\Gamma)$ фононов) обертонами поперечных $2 \mathrm{TO}(\Sigma)$ фононов при $662 \mathrm{~cm}^{-1}, 2 \mathrm{TO}(\mathrm{K})$ фононов 


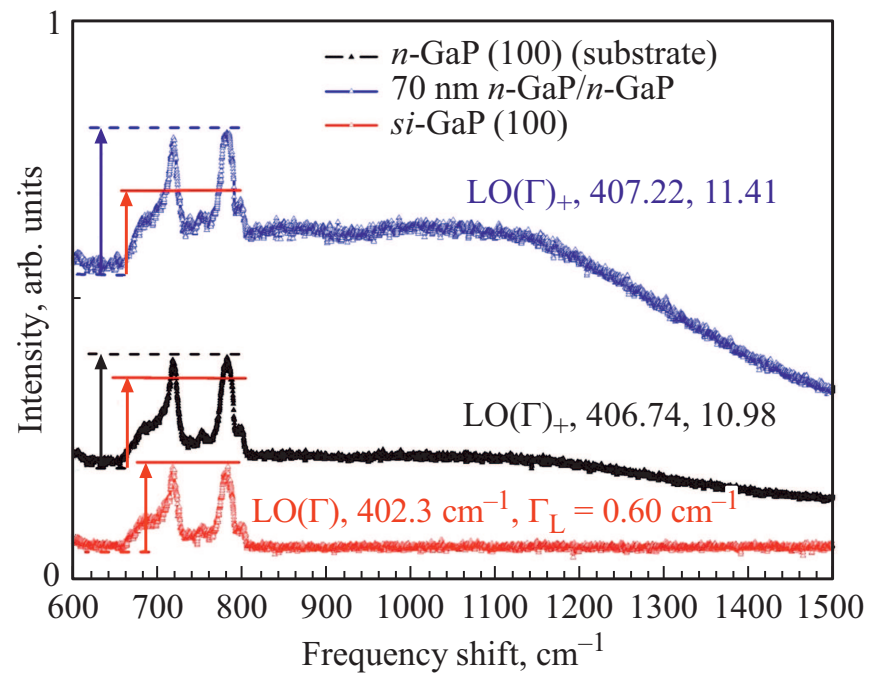

Спекры рамановского рассеяния света второго порядка оптическими фононами и фотолюминесценции в диапазоне частот $600-1500 \mathrm{~cm}^{-1}$ в кристаллах (001) si-GP (нижний), (001) $n$-GaP (средний) и образце $n$ - $\mathrm{GaP} / n-\mathrm{GaP}$ с гомоэпитаксиальным нанослоем $n$-GaP (верхний). Интенсивности спектров рассеяния света второго порядка, оптическими фононами для всех приведенных спектров указанны соответствующими вертикальными стрелками.

Спектры получены при комнатной температуре и при возбуждении излучением лазера с длиной волны $\lambda_{i}=532.070 \mathrm{~nm}$. Толщина нанослоя, выращенного методом газофазной эпитаксии из металлоорганических соединений на проводящей подложке $n$-GaP, ориентированной по оси (001), равна $70 \mathrm{~nm}$. Указанные величины значения частоты и полуширин линий (полных ширин на половине интенсивости) рамановского рассеяния света первого порядка поперечными $\mathrm{TO}(\Gamma)-$ и продольными LO(Г) оптическими фононами, а также высокочастотными продольными связанными плазмон-фононными $\mathrm{LO}(\Gamma)_{+}$ колебаниями полученны с выделением лоренцевого контура из наблюдаемого фохтовского контура с учетом поправки на спектральное разрешение.

при $682 \mathrm{~cm}^{-1}, 2 \mathrm{TO}(\mathrm{X})$ фононов при $696 \mathrm{~cm}^{-1}, 2 \mathrm{TO}(\mathrm{L})$ фононов при $720 \mathrm{~cm}^{-1}$ и продольных $2 \mathrm{LO}(\mathrm{L}, \mathrm{X})$ фононов при $783 \mathrm{~cm}^{-1}$ и поперечных $2 \mathrm{TO}(\Gamma)$ фононов при $805 \mathrm{~cm}^{-1}$, а также суммарными комбинациями продольных и поперечных оптических $\mathrm{LO}(\mathrm{X})+\mathrm{TO}(\mathrm{X})$ фононов при $738 \mathrm{~cm}^{-1}$, и $\mathrm{LO}(\mathrm{L})+\mathrm{TO}(\mathrm{L})$ фононов при $734 \mathrm{~cm}^{-1}$, с волновыми векторами соответствующими точкам $\Sigma$, $\mathrm{K}, \mathrm{X}, \mathrm{L}$ и Г зоны Бриллюэна кристалла GaP. Можно отметить четкое различие этих спектров по интенсивности линий двухфононного рассеяния и широкой полосы фотолюминесценции, непосредственно примыкающей к линиям двухфононного рассеяния. Для сравнения интенсивности спектров рассеяния света второго порядка, оптическими фононами для всех приведенных спектров указаны соответствующими вертикальными стрелками. Хорошо видно, что при оптическом возбуждении с использованием линии лазера на алюмоиттриевом гранате с энергией квантов возбуждающего излучения $\hbar \omega_{i}=2.33 \mathrm{eV}$ выше непрямого края поглощения $n-\mathrm{GaP}$
$E_{g}=2.27 \mathrm{eV}$ рассеяние света для легированного гомоэпитаксиального слоя $n$-GaP (001) в сравнении со спектром для полуизолирующего кристалла $s i-\mathrm{GaP}$ (001) носит резонансный характер. Аналогичное, но несколько меньшее резонансное усиление обнаруживается и для легированной подложки кристалла $n$-GaP $(001)$ в образце $n-\mathrm{GaP} / n-\mathrm{GaP}(001)$. При этом меньшая величина резонансного усиления хорошо согласуется с меньшей величиной интенсивности рекомбинационного излучения экситона. Обнаруженное усиление обусловлено возможным присутствием примесей азота, интересным образом подтверждаемое выявлением дополнительных широких полос фотолюминесценции. Они хорошо заметны в спектрах, приведенных на рис. 1 для гомоэпитаксиального слоя $n$-GaP (001) и сильнолегированной подложки $n-\mathrm{GaP}(001)$ в образце $n-\mathrm{GaP} / n-\mathrm{GaP}$ (001). Обнаружение таких полос фотолюминесценции связано с квазипрямыми экситонами образованными локализованными состояниями наиболее близких пар атомов азота, при их небольшой концентрации $[\mathrm{N}] \sim 10^{16} \mathrm{~cm}^{-3}$. Тогда резонансный характер усиления интенсивностей спектров рассеяния света второго порядка оптическими фононами может быть обусловлен проявлением экситонфононного взаимодействия [15-23]. Оно обусловлено возможным присутствием примесей азота, существенно модифицирующих энергетический спектр экситонов с образованиием квазипрямых экситонов [44-48], образованных локализованными состояниями наиболее близких пар атомов азота.

\section{4. Заключение}

В настоящей работе показано, что на основе развития методики спектроскопии микрорамановского резонансного рассеяния света в гомоэпитаксиальном наномасштабном слое легированного кристалла $n$-GaP, толщиной $70 \mathrm{~nm}$, выращенном на сильнолегированной подложке кристалла $n-\mathrm{GaP}$, ориентированной по оси $(001)$ в спектре рамановского рассеяние света в диапазоне частот от 600 до $800 \mathrm{~cm}^{-1}$ удается обнаружить достаточно узкие полосы линий второго порядка при комнатной температуре. Такие характерные линии зарегистрированы и в спектрах для сильнолегированной подложки $n$ - $\mathrm{GaP}$ в образце $n-\mathrm{GaP} / n-\mathrm{GaP}(001)$ и в отдельном образце полуизолирующего кристалла $s i-\mathrm{GaP}(001)$. Установлено, что такое рассеяние возможно обусловлено обычно слабыми суммарными комбинациями и обертонами поперечных $\mathrm{TO}(\Gamma)$ и продольных $\mathrm{LO}(\Gamma)$ оптических фононов, с волновыми векторами соответствующими точкам $\Sigma, \mathrm{K}, \mathrm{X}, \mathrm{L}$ и $Г$ зоны Бриллюэна кристалла GaP при оптическом возбуждении вблизи непрямого края поглощения легированного гомоэпитаксиального слоя $n$-GaP. Показано, что рассеяние света гомоэпитаксиального слоя $n$-GaP носит резонансный характер вследствие проявления экситон-фононного взаимодействия. Оно обусловлено возможным присутствием примесей азота, выявленных по спектрам фотолюминесценции, 
когда существенно модифицируется реальный энергетический спектр экситонов с образованием квази-прямых экситонов, образованных локализованными состояниями наиболее близких пар атомов азота.

\section{Конфликт интересов}

Автор заявляет об отсутствии конфликта интересов.

\section{Список литературы}

[1] M. Itoh. Prog. Surf. Sci. 66, 53 (2001).

[2] P. Kratzer, C.G. Morgan, M. Scheffler. Phys. Rev. B 59, 15246 (1999).

[3] Ю.Г. Галицын, Д.В. Дмитриев, В.Г. Мансуров, С.П. Мощенко, А.И. Торопов. Письма в ЖЭТФ 86, 553 (2007).

[4] O.A. Ageev, M. Solodovnik, S.V. Balakirev, I.A. Mikhalin, M. Eremenko. J. Cryst. Growth 457, 46 (2017).

[5] О.С. Комков, А.Н. Пихтин, Ю.В. Жиляев, Л.М. Федоров. Письма в ЖТФ 34, 1, 81 (2008).

[6] B. Németh, W. Kunert, K. Stolz, K. Volz. J. Cryst. Growth. 310, 1595 (2008).

[7] S. Nagarajan, H. Jussila, J. Lemettinen, K. Banerjee, M. Sopanen, H. Lipsanen. J. Phys. D 46, 165103 (2013).

[8] О.С. Комков, Д.Д. Фирсов, Т.В. Львова, И.В. Седова, А.Н. Семенов, В.А. Соловьев, С.В. Иванов. ФТТ 58, 707 (2016).

[9] K. Storm, F. Halvardsson, M. Heurlin, D. Lingren, A. Gustafsson, P.M. Wu, B. Monemar, L. Samuelson. Nature Nanotechnol. 7, 718 (2012).

[10] Light Scattering in Solids. Topics in Applied Physic / Ed. M. Cardona, G. Guntherodt. Sringer, Berlin, Heidelberg, N.Y. (1974). $543 \mathrm{p}$.

[11] R. Loudon. Proc. R. Soc. Lond. A 275, 218 (1963).

[12] A.K. Ganguly, J.L. Birman. Phys. Rev. B 162, 806 (1967).

[13] R.M. Martin. Phys. Rev. B 4, 3676 (1971).

[14] R. Zeyher, T. Chiu-Sen, J.L. Birman. Phys. Rev. B 4, 1725 (1974).

[15] Б.Х. Байрамов, А.В. Гольцев, Э. Карайамаки, Р. Лайхо, Т. Левола, В.В. Топоров. ФТТ 25, 1286 (1983).

[16] E. Karajamaki, R. Laiho, T. Levola, B.H. Bairamov, A.B. Gol'tsev, V.V. Toporov. Phys. Rev. B 29, 4508 (1984).

[17] B.H. Bairamov, A.V. Gol'sev, V.V. Toporov, L. Laiho, T. Levola. Proc. of the 18th Int. Conf. on Phys. of Semicond / Ed. O. Engstrom, World Scientific, Singapure (1987). P. 17211724.

[18] Б.Х. Байрамов, Н.В. Личкова, А.В. Гольцев, В.Д. Тимофеев, В.В. Топоров. ФТТ 29, 244 (1987).

[19] A. Cantarero, C. Trallero-Giner, M. Cardona. Phys. Rev. B 39, 8388 (1989).

[20] C. Trallero-Giner, A. Cantarero, M. Cardona. Phys. Rev. B 40, 4030 (1989).

[21] C. Trallero-Giner, A. Cantarero, M. Cardona. Phys. Rev. B 40, 12290 (1989).

[22] A. García-Cristóbal, A. Cantarero, C. Trallero-Giner, M. Cardona. Phys. Rev. B 49, 13430 (1994).

[23] C. Trallero-Giner, K. Syassen. Phys. Status Solidi B 247, 182 (2010).

[24] R.P. Miranda, M.I. Vasilevskiy, C. Trallero-Giner. Phys. Rev. B 74, 115317 (2006).
[25] C. Trallero-Giner, K. Kunc, K. Syassen. Phys. Rev. B 73, 205202 (2006).

[26] A.M. Yaremko, V.V. Koroteev, V.O. Yukhymchuk, V.M. Dzhagan, H. Ratajczak, A.J. Barnes, B. Silvi. Chem. Phys. 388, 57 (2011).

[27] Ф.Б. Байрамов, В.В. Топоров, Е.Д. Полоскин, Б.Х. Байрамов, C. Röder, C. Sprung, C. Bohmhammel, G. Seidel, G. Irmer, A. Lashkul, E. Lahderanta, Y.W. Song. ФТП 47, 607 (2013).

[28] Б.Х. Байрамов. ФТТ 58, 707 (2016). Phys. Solid State 58, 728 (2016).

[29] L.G. Cançado, A. Jorio, M.A. Pimenta. Phys. Rev. B 76, 064304 (2007).

[30] A.G. Rolo, M.I. Vasilevskiy, M. Hamma, C. Trallero-Giner. Phys. Rev. B 78, 081304(R) (2008).

[31] Ф.Б. Байрамов, Е.Д. Полоскин, А.Л. Чернев, В.В. Топоров, М.В. Дубина, Б.Х. Байрамов. ФТП 51, 10, 1376 (2017)

[32] B. Carvalho, Y. Wang, S. Mignuzzi, L.M. Malard, M.A. Pimenta. Nature Commun. 8, 14670 (2017).

[33] Ф.Б. Байрамов, Е.Д. Полоскин, А.Л. Чернев, В.В. Топоров, М.В. Дубина, С. Sprung, Н.К. Lipsanen, Б.Х. Байрамов. Письма ЖТФ, 44, 2, 72 (2018).

[34] G. Gordeev, B. Flavel, R. Krupke, P. Kusch, S. Reich. Phys. Rev. B 99, 045404 (2019).

[35] Ф.Б. Байрамов, В.В. Топоров, Е.Д. Полоскин, Б.Х. Байрамов, М.В. Дубина, Е. Лахдеранта, Х. Липсанен, Б.Х. Байрамов. Письма в ЖЭТФ 99, 437 (2014).

[36] Y. Wang, B.R. Carvalho, V.H. Crespi. Phys. Rev. B 98, 161405(R) (2018).

[37] J.T. Holmi, B.H. Bairamov, S. Suihkonen H. Lipsanen. J. Cryst. Growth 499, 47 (2018).

[38] B.H. Bairamov, V.V. Toporov, F.B. Bayramov, A.D. Bouravleuv, J.T. Holmi, H. Lipsanen, V.P. Popov, I.N. Kuprianov, Yu.N. Palánov, D. Braukmann, J. Debus, D.R. Yakovlev, М. Вayer. Сибир. физ. журн. 13, 3, 73 (2018).

[39] B.H. Bairamov, V.V. Toporov, F.B. Bayramov. ФТП 53, 85 (2019).

[40] Б.Х. Байрамов, В.В. Топоров, В.Н. Вишневский, М.И. Демчук, В.В. Топоров, Ш.Б. Убайдуллаев, Л. Хилдиш, Э. Яне. ФTT 23, 14 (1981).

[41] B.H. Bairamov, V.V. Toporov, F.B. Bayramov. ФTT 63, 80 (2021).

[42] G. Irmer, V.V. Toporov, B.H. Bairamov, J. Monecke. Phys. Status Solidi 119, 2, 595 (1983).

[43] B.H. Bairamov A. Heinrich, G. Irmer, V.V. Toporov, E. Ziegler. Phys. Status Solidi 119, 1, 227 (1983).

[44] В.С. Горелик, А.Л. Карузский, П.П. Свербиль. ФТТ 44, 1770 (2002).

[45] H.P. Xin, C.W. Tu, Y. Zhang, A. Mascarenhas. APL 76, 1276 (2000).

[46] W. Shan, W. Walukiewicz, K.M. Yu, J. Wu, H.P. Xin, Y.G. Hong, C.W. Tu. Appl. Phys. Lett. 76, 3251 (2000).

[47] P.R.C. Kent, A. Zunger. Phys. Rev. B 64, 115208 (2000).

[48] I.A. Buyanova, G. Pozina, J.P. Bergman, W.M. Chen, H.P. Xin, C.W. Tu. Appl. Phys. Lett. 81, 52 (2002).

Редактор Т.Н. Василевская 